INTRODUCTION

\title{
Youth tobacco use research in the USA: progress and challenges
}

\section{T J Glynn, S L Mills}

Tobacco Control 2003;12(Suppl IV):iv1-iv2

forts to control tobacco use and tobacco related morbidity and mortality in the USA have met with reasonable success, at least through the early part of the 1990s. In the 39 years since the publication of the first Surgeon General's Report on Tobacco and Health, adult smoking rates in the USA have declined by nearly $40 \%$, although only a modest portion of that decline has occurred during the past decade. Nevertheless, less than a quarter of the US population now smokes and other indices of success, such as changing attitudes toward tobacco use in public places, a near universal perception of tobacco use as a health hazard, and support for laws and regulations restricting tobacco use all point to continued progress in tobacco control among the US adult population.

Controlling tobacco use among youth, however, has presented a greater challenge in recent years. Although there was considerable progress in reducing adolescent tobacco use between the early 1970s and the mid 1980s, incidence and prevalence of youth smoking began, first, to stabilise in the late 1980s and, then, alarmingly, to climb through a large portion of the 1990s. In fact, in the relatively short period between 1991 and 1997, the percentage of US high school students who were current smokers rose by nearly a third, from $27 \%$ to $36 \%$.

The research community offered a number of potential explanations for this reversal of fortune in youth tobacco use-the tobacco industry's growing aggressiveness in targeting youth audiences (best personified by the omnipresence of the Joe Camel character); the possibility that the youth smoking prevention programmes created in the 1970s and '80s were simply not as effective in deterring a new generation of youthful tobacco experimenters from becoming regular users; the increase in smokeless tobacco use and new, flavoured products in the 1980s that may have provided an entrée for many youth, particularly males, to begin smoking; the growing diversity of the US population and subsequent volatility in tobacco use among subgroups of youth, such as the wide range of prevalence rates among African American adolescent males during the 1990s and the divergence in smoking rates between Latino adolescent males and females; the relatively low cost of cigarettes for youth, due to an expanding economy and tobacco excise taxes which had not risen with the economy; the increase in tobacco use in a variety of media aimed at youth; as well as numerous others.

\section{"A PEDIATRIC DISEASE"}

Further, the then Food and Drug Administration commissioner Dr David Kessler, had, in 1995, termed youth tobacco use "a pediatric disease", thereby inviting the medical community to become more involved in this issue and instigating a wider public debate about the topic.

It was in this context that the National Cancer Institute (NCI), along with several of its sister National Institutes of Health (NIH) institutes, began, in 1996, to develop a research plan which would address some of the reasons for the sharp increase in youth tobacco use that the USA was then experiencing and begin to provide a solid base of youth tobacco research which would serve the public health community well into the new millennium. This research planning process culminated in the issuance of Requests for Applications (RFAs) in 1997 and 1999 entitled "Prevention and cessation of tobacco use by children and youth".

There were several unique aspects to these RFAs. First, they were the first NIH research programme to focus exclusively on the youth tobacco problem. Second, they represented an alliance of NIH institutes, involving not only the NCI, but also the National Institute on Drug Abuse, National Institute of Child Health and Human Development, the National Institute of Dental and Craniofacial Research, and the National Institute of Nursing Research.

Finally, they approached the youth tobacco problem from a multidisciplinary, multipronged perspective, inviting a range of disciplines-from anthropology to genetics to neurology-and areas of expertise-from policy analysts to clinicians to economists-to focus their talents and experience on this issue.

\section{ACCOMPLISHMENTS TO DATE}

What has been accomplished thus far? The NCI and its NIH collaborators have amassed an array of more than 30 high quality, major research trials with the single aim of developing strategies for the immediate and significant reduction of tobacco use by children and youth in the USA; have helped create and nurture a cadre of youth tobacco researchers who will be making contributions to this field-domestically and internationally-for many years to come; have

Abbreviations: $\mathrm{NCl}$, National Cancer Institute; $\mathrm{NIH}$, National Institutes of Health; RFA, Requests for Applications 
provided a training ground for a new generation of youth tobacco researchers; have demonstrated the importance of utilising a range of investigative disciplines when focusing on a single issue; have initiated or participated in a number of collaborations and alliances with other federal government agencies, states, foundations, and international organisations; and instituted a widely attended annual research conference on youth tobacco use which has helped focus national and international research attention on this topic.

The articles in this special supplement to Tobacco Control represent a cross section of the exceptional work that has been done thus far through this initiative. This special issue is not a culmination of this process, but merely represents a snapshot of what has been learned from this research initiative to date, as well as a tantalising glimpse into the future. Ranging from the development and testing of new measurement techniques with adolescent smokers to a new look at why youths start smoking in the first place to studies which address the exceptionally difficult problem of treating nicotine dependence in its early stages, the studies in this volume demonstrate the promise of the entire body of research which is being supported through this collaborative enterprise.

The articles in this supplement then, as well as the data and manuscripts emerging from many of the other teams involved in this singular endeavour, provide a research roadmap for the future-the need to develop new and practical cessation methods for youthful smokers; to consider the controversial issue of harm reduction as it may apply to adolescents; to investigate continuing effects of media on youth smoking uptake and maintenance; to re-explore the effectiveness of educational programmes aimed at youth tobacco use prevention; to consider genetic predispositions to nicotine dependence in youth, and the ethical issues this may raise; to examine the rising potential of policy change as a tobacco control tool for youth, etc. An essential direction of this roadmap will be, in addition to the specific research needs above, a clear call for the widespread dissemination and adoption of the results obtained from this research.

If the NCI and its collaborators can continue to harness the same energy, creativity, and research acumen that they have done thus far in this initiative-as represented by the innovative articles in this issue-then the future for youth tobacco research and, more importantly, the eventual reduction and elimination of youth tobacco use, will be bright indeed.

Authors' affiliations

T J Glynn, American Cancer Society, Washington DC, USA

S L Mills, Abt Associates, Bethesda, Maryland, USA 